\title{
Risk Factors for Hospital-Acquired Pneumonia in Hemorrhagic Stroke Patients Treated in the Intensive Care Unit
}

\author{
Sol Ji Jung ${ }^{1}$, Ja Myoung Lee ${ }^{2}$, Tae yong An ${ }^{1}$, In Sung Park ${ }^{3}$ \\ ${ }^{1}$ Department of Neurosurgery, Gyeongsang National University Hospital, Gyeongsang National University School of Medicine, Jinju, Korea \\ ${ }^{2}$ Department of Neurosurgery, Gyeongsang National University School of Medicine and Gyeongsang National University Changwon Hospital, Changwon, \\ Korea \\ ${ }^{3}$ Departement of Neurosurgery, Changwon Hanmaeum Hospital, Changwon, Korea
}

Received: September 9, 2021

Accepted: September 22, 2021

Corresponding Author:

Ja Myoung Lee, M.D. Ph.D.

Department of Neurosurgery, Gyeongsang National University School of Medicine and Gyeongsang National University Changwon Hospital, 11

Samjeongja-ro, Seongsan-gu, Changwon-si, Gyeongsangnamdo, Korea

Tel : +82-55-214-2440

Fax : +82-55-214-1031

E-mail : wkauddl82@naver.com

\section{Objective}

Pneumonia is a very serious medical complication in patients with hemorrhagic stroke such as spontaneous intracerebral hemorrhage and subarachnoid hemorrhage. In the case of hemorrhagic stroke patients, hospital-acquired pneumonia increases morbidity, mortality and medical costs in addition to the already poor prognosis of hemorrhagic stroke. The purpose of this study was to identify risk factors for hospital-acquired pneumonia in hemorrhagic stroke patients treated in the intensive care unit.

\section{Methods}

Our study was a retrospective review of 112 hemorrhagic stroke patients treated in an intensive care unit who were hospitalized in the neurosurgery department of Gyeonsang National University Hospital from August 2019 to July 2020. The data included basic demographic data, the underlying disease, lifestyle factors, neurological evaluation results, severity of the condition and other characteristics. The radiological data and medical records of the patients were retrospectively analyzed.

Results

A total of 97 patients were included in the study, and 10 of them met the diagnostic criteria for hospital-acquired pneumonia. Diabetes mellitus, a high simplified acute physiology score 3 (SAPS3), a low glasgow coma scale (GCS) score, mechanical ventilation, tracheostomy, dysphagia and nasogastric tube feeding were identified as risk factors for the development of hospital-acquired pneumonia $(\mathrm{p}<0.05)$. Six of 10 bacterial pathogens isolated from sputum were identified as multidrug-resistant pathogens. Hospital-acquired pneumonia led to further antibiotic treatment and general deterioration, which in turn increased the intensive care unit length of stay $(\mathrm{p}<0.001)$. Conclusion

We found that mechanical ventilation, tracheostomy, dysphagia, tube feeding, a high SAPS3, and a low GCS score were risk factors for hospital-acquired pneumonia (HAP) in hemorrhagic stroke patients. Efforts will be needed to prevent pneumonia by understanding the risk factors for HAP identified in our study.

Keywords: Hospital - acquired pneumonia; Hemorrhagic stroke; Cerebral hemorrhage; Subarachnoid hemorrhage 


\section{INTRODUCTION}

Hemorrhagic stroke, including spontaneous intracerebral hemorrhage and subarachnoid hemorrhage, accounts for 10 to $20 \%$ of all strokes ${ }^{1,2}$. The mortality risk is highest in the first week after a hemorrhagic stroke, and the overall patient mortality rate for the first month is reported to be approximately $40 \%$ for intracerebral hemorrhage and approximately $30 \%$ for subarachnoid hemorrhage ${ }^{1)}$. For this reason, it is essential that patients with hemorrhagic stroke are treated in the intensive care unit (ICU), and it is important to understand the associated medical complications and focus on prevention and treatment. Of the many complications, chest infection is the most common medical complication of acute stroke. Hospital-acquired pneumonia (HAP) is the most common infection in the ICU. This infection encompasses two different entities: pneumonia associated with mechanical ventilation and severe pneumonia developed during a hospital stay ${ }^{3,4}$.

Patients with hemorrhagic stroke are generally exposed to the risk of nosocomial infection, and respiratory infections account for 30 to $60 \%$ of all infections in the ICU. The incidence of pneumonia in the ICU was reported to range from $10 \%$ to $60 \%$, currence of pneumonia increases the duration of the ICU stay, mortality rate, and associated health care costs. In addition, the risk of delayed neurological deficits in patients is increased by the fever that accompanies the infection and aggravates functional disability and cognitive impairments ${ }^{7,8)}$.

In hemorrhagic stroke patients, pneumonia can be seen as an extension of the treatment of hemorrhagic stroke that is probably unavoidable, and the control of pneumonia is closely related to patient prognosis. Several studies have been conducted to identify risk factors that increase the incidence of pneumonia in hemorrhagic stroke patients. Accurately recognizing these risk factors and focusing on treatment will be helpful in patient treatment and prognosis assessment.

To understand the risk factors associated with hospital-acquired pneumonia after hemorrhagic stroke and benefit from more active treatment of at-risk patients, we retrospectively evaluated $112 \mathrm{hem}$ orrhagic stroke patients in the ICU.

\section{MATERIALS AND METHODS}

\section{Patient Selection and Study Design}

Our study was conducted through retrospective chart reviews of 112 hemorrhagic stroke patients who were admitted to the Gyeongsang National University Hospital between August 2019 and July 2020. Fifteen of the 112 patients were excluded for the following reasons: five people had pneumonia at the time of hospitaliza- tion, seven people died of brain herniation and other causes within three days of hospitalization, and three people were transferred to other hospitals (Fig. 1).

The diagnosis of pneumonia was based on American Thoracic Society Guidelines, which are as follows. HAP is nosocomial pneumonia that occurs 48 hours after admission, and ventilator-associated pneumonia (VAP) is pneumonia that occurs 48 hours to 72 hours after intubation. Regardless of the presence of progressive hypoxemia, patients with two or more clinical signs of lung infection, such as fever, purulent sputum, and leukocytosis, can be diagnosed with HAPif new or progressive radiographic infiltrates are seen on chest X-ray ${ }^{9}$.

\section{Variables and Data Collection}

The radiological data and medical records of the patients were retrospectively analyzed. In addition to basic demographic data (age and sex), the following categories of data were collected for each patient: underlying disease (hypertension, diabetes mellitus, and dyslipidemia), lifestyle factors (smoking and alcohol use), neurological evaluation and severity of condition at the time of the patient's hospitalization and upon discharge from the ICU [Glasgow Coma Scale (GCS), Glasgow Outcome Scale (GOS), the Simplified Acute Physiology Score 3 (SAPS3), and ICU stay period], and invasive treatments and procedures (ventilator application and tracheostomy). Finally, we collected data on dysphagia and tube feeding, which are thought to be common complications of hemorrhagic stroke patients.

\section{Statistical Analysis}

Categorical variables are presented as the number, and continuous variables are presented as the mean $\pm \mathrm{SD}$. Comparisons of continuous variables between groups were made by independent sample t-tests. For the comparison of categorical variables between groups, the chi-square test or Fisher's exact test was used when ap-

$$
112 \text { patients were admitted }
$$
$(2019 / 08 / 01-2020 / 07 / 31)$

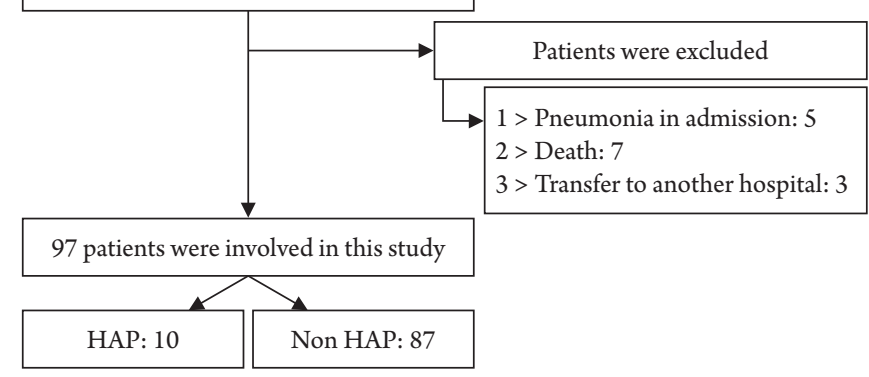

Fig. 1. Patient selection of the study. HAP: Hospital-acquired pneumonia 
propriate. The significance level was set at $\mathrm{p}=0.05$. The statistical software used for analyses was SPSS for Windows software (SPSS 21.0, IBM, Inc., NY).

\section{RESULTS}

\section{Patient Characteristics}

A total of 97 patients were included in the study, and 10 of them met the diagnostic criteria for hospital-acquired pneumonia. All 10 patients were diagnosed with pneumonia by pulmonology specialists at Gyeongsang National University Hospital; 6 of the patients were male, and 4 were female. The mean age of the patients with pneumonia was $62.9 \pm 12.8$, compared to $57.9 \pm 13.3$ for those who did not experience pneumonia.

Among the patients without pneumonia, 48 patients had hypertension, 20 patients had diabetes mellitus, and 10 patients had a history of dyslipidemia. Of 26 diabetes mellitus patients, 6 patients had pneumonia $(p=0.02)$. There were 26 smokers in the study, of whom 3 had pneumonia. Of the 87 patients without pneumonia, $29.8 \%$ (26) consumed alcohol compared to $20 \%$ (2) of the patients with pneumonia.

In this study, we describe the severity of the condition of each patient with the SAPS3, GCS, GOS, and length of ICU stay. The SAPS3 of patients with and without pneumonia were $48 \pm 12.7$ and $36.9 \pm 10.9$, respectively. Patients with pneumonia had lower
GCS scores at admission and lower GOS scores at discharge. Patients with pneumonia showed an average GCS score of 7.2 \pm 3.52 and GOS score of $2.8 \pm 0.9$, and patients without pneumonia showed an average GCS score of $12.88 \pm 2.48$ and $3.9 \pm 0.9$. As a result, the length of ICU stay of patients with pneumonia was $28 \pm$ 10.2 days, which is 2 times longer than that of patients who did not have pneumonia $\left(10.0 \pm 9 .^{1)}\right.$. All parameters of the severity of the patient's neurologic condition that we set were significantly correlated with pneumonia $(\mathrm{p}<0.05)$.

A total of 32 patients underwent mechanical ventilation, including 9 of the 10 patients with pneumonia (90\%) and 23 of the 87 patients without pneumonia (26.4\%). Additionally, 9 tracheostomies were performed in the pneumonia group, compared to 12 tracheostomies in the group of patients without pneumonia. Factors associated with the risk of pneumonia were tube feeding ( 9 of 10 patients with pneumonia, 31 of 87 patients without pneumonia) and dysphagia ( 9 of 10 patients with pneumonia, 27 of 87 patients without pneumonia). All of the factors are described in Table 1.

\section{DISCUSSION}

HAP is the most common infection in the intensive care unit. This infection comprises two different entities: pneumonia related to mechanical ventilation and severe pneumonia devel-

Table 1. Characteristics of the patients with pneumonia and without pneumonia

\begin{tabular}{|c|c|c|c|}
\hline Characters & Patients with pneumonia $(n=10)$ & Patients without pneumonia $(\mathrm{n}=87)$ & pvalue \\
\hline \multicolumn{4}{|l|}{ Basic demographic data } \\
\hline M & 6 & 28 & $0.13^{*}$ \\
\hline $\mathrm{F}$ & 4 & 54 & $0.22^{*}$ \\
\hline Age (years) & $57.9 \pm 13.3$ & $62.9 \pm 12.8$ & 0.87 \\
\hline \multicolumn{4}{|l|}{ Underlying disease } \\
\hline Hypertension & 9 & 48 & $0.92^{*}$ \\
\hline \multicolumn{4}{|l|}{ Life style } \\
\hline Smoking & 3 & 23 & $0.89^{*}$ \\
\hline Alcohol use & 2 & 26 & $0.49^{*}$ \\
\hline \multicolumn{4}{|c|}{ Neurological evaluation \& severity of condition } \\
\hline SAPS3 & $48 \pm 12.7$ & $39.6 \pm 10.9$ & 0.02 \\
\hline \multicolumn{4}{|l|}{ Other characteristics } \\
\hline Mechanical ventilator & 9 & 23 & $<0.001^{*}$ \\
\hline Tracheostomy & 9 & 12 & $<0.001^{*}$ \\
\hline Tube feeding & 9 & 31 & $0.002^{*}$ \\
\hline Dysphagia & 9 & 27 & $<0.001^{*}$ \\
\hline
\end{tabular}

SAPS3: Simplified acute physiology score 3, GCS: Glasgow coma scale, GOS: Glasgow outcome score.

${ }^{*}$ Calculated using Fisher's exact test; the chi-square test was used for other calculations to compare the categorical variables between groups. 
oped during the hospital stay. VAP continues to complicate the course of 8 to $28 \%$ of patients receiving mechanical ventilation. In the ICU, HAP is associated with an approximate mortality rate of $20 \%$. Hemorrhagic stroke is a severe disease that requires intensive care and causes several medical complications, the most common of which is pneumonia ${ }^{10,11}$. We conducted a retrospective study on pneumonia that occurred during ICU care and its risk factors in hemorrhagic stroke patients and identified several significant factors.

Patients treated with a ventilator due to respiratory failure or hyperventilation to control increasing intracranial pressure had a high risk of airway bacterial colonization and lung infection. The mechanism suggested by LaForce was colonization of the lower respiratory tract with microorganisms, continuous microaspiration, and overcoming of lung defense mechanisms. For this reason, VAP is strongly associated with the duration of mechanical ventila$\operatorname{tion}^{12,13)}$. The subsequent need for tracheostomy in some patients who require prolonged use of mechanical ventilation significantly increases the risk of pneumonia. Patients with a tracheostomy are at increased risk of ventilator-associated tracheobronchitis, which is usually a precursor to VAP. Bronchial colonization by bacteria in the upper airways during the tracheostomy procedure represents a reservoir for lower airway colonization and increases the risk of pneumonia ${ }^{14)}$.

Dysphagia is a common complication of stroke, but estimates of its frequency vary considerably. It is a significant cause of pneumonia on the first day after stroke, and previous studies have reported an increased risk of mortality in the acute phase. In addition, dysphagia has been shown to be associated with malnutrition, dehydration and an increased length of hospital stay ${ }^{15)}$. Patients with dysphagia had a much higher risk for pneumonia than those with normal swallowing, which could lead to higher hospitalization costs. This finding is consistent with a review that showed a 3-fold increased risk of pneumonia among patients with dysphagia and an 11-fold increased risk among those with aspiration ${ }^{16)}$. In addition, the use of tube feeding was associated with an increased risk of pneumonia in our study. Whether the placement of a nasogastric tube further increases the risk of pneumonia by promoting colonization of the oropharynx with pathogenic bacteria is still controversial, as most aspiration pneumonia has a bacterial origin. However, one important problem is that the feeding tube does not prevent the aspiration of gastroesophageal reflux ${ }^{17}$. A retrospective review indicated nasogastric tubes and immobility to be stronger predictors for respiratory infections than dysphagia in acute stroke ${ }^{18)}$.

Diabetic subjects may have increased susceptibility to pneumonia for several reasons. They are at increased risk of aspiration, hy- perglycemia, decreased immunity, impaired lung function, pulmonary microangiopathy, and coexisting morbidities ${ }^{19)}$. Microvascular changes in the basement membranes of pulmonary blood vessels and the respiratory epithelium in diabetic patients increase the risk of pneumonia-related hospitalization ${ }^{20)}$. Therefore, strict glycemic control is important for diabetic subjects to prevent pneumonia. In addition, the worse the neurologic status was, the more frequent the occurrence of HAP, which resulted in a longer ICU length of stay. Thus, HAP increases the duration of hospital stay and affects morbidity and outcomes. For this reason, early antibiotics for treating HAP should be selected carefully, because HAP increases ICU mortality and costs.

In patients at high risk of infection caused by multidrug-resistant bacteria, combined antibiotic therapy is performed from the beginning. Empirical antibiotics should be adjusted after antibiotic resistance evaluation based on the results of the sputum culture. In one prospective study, $45 \%$ of patients with HAP presented with multidrug-resistant organisms ${ }^{21}$. Our study also found that six of the 10 microorganisms identified in the sputum culture showed multidrug resistance (Table 2). The knowledge of local bacterial flora and resistance patterns is of crucial importance and is strongly recommended. This evidence increases the probability of success of empiric antibiotic therapy.

Like any retrospective study, our study has several limitations. Selection bias can exist, as this was a retrospective study in a single center. The variables did not include the size and location of the hemorrhage. In addition, our study is limited in that it is a small case study and the results may not be generalized.

\section{CONCLUSION}

ICU care is essential for patients with hemorrhagic stroke. A major medical complication of hemorrhagic stroke is hospital-acquired pneumonia. We analyzed the risk factors for HAP

Table 2. Bacterial pathogens isolated from sputum

\begin{tabular}{ll}
\hline Hospital day with ventilator & Sputum Culture Findings \\
\hline Casel. Do not apply ventilator & K.Pneumoniae ESBL ${ }^{+*}$ \\
Case2. 11th day of hospitalization & K.Pneumoniae ESBL ${ }^{+*}$ \\
Case3. 4th day of hospitalization & Acinetobacter nosocomialis* \\
Case4, 4th day of hospitalization & Streptococcus pneumoniae \\
Case5. 4th day of hospitalization & Haemophilus influenza \\
Case6. 4th day of hospitalization & Enterobacter cloacae complex \\
Case7. 6th day of hospitalization & Haemophilus influenza \\
Case8. 14th day of hospitalization & Pseudomonas. Aeruginosa* \\
Case9.9th day of hospitalization & Pseudomonas. Aeruginosa* \\
Case 10, 4th day of hospitalization & K.Pneumoniae ESBL \\
\hline${ }^{*}$ MDR: Multidrug resistance & \\
${ }^{+}$Extended-spectrum beta-lactamase &
\end{tabular}

Hospital day with ventilator K.Pneumoniae ESBL ${ }^{+*}$ Haemophilus influenza Enterobacter cloacae complex Haemophilus influenza Pseudomonas. Aeruginosa* Pseudomonas. Aeruginosa Case 10, 4th day of hospitalization ${ }^{\dagger}$ Extended-spectrum beta-lactamase 
in hemorrhagic stroke patients and found that mechanical ventilation, tracheostomy, dysphagia, tube feeding, a high SAPS3 score, and a low GCS score were risk factors for HAP.

In addition, multidrug-resistant pathogen infection is common, so caution is required during antibiotic selection by referring to sputum culture results and antibiotic sensitivity tests. Efforts are needed to prevent pneumonia by understanding the risk factors for HAP identified in our study.

\section{NOTES}

\section{Conflict of interest}

No potential conflict of interest relevant to this article was reported.

\section{REFERENCES}

1. González-Pérez A, Gaist D, Wallander MA, McFeat G, García-Rodríguez LA. Mortality after hemorrhagic stroke: data from general practice (The Health Improvement Network). Neurology 2013;81:559-65.

2. Sudlow CL, Warlow CP. Comparable studies of the incidence of stroke and its pathological types: results from an international collaboration. International Stroke Incidence Collaboration. Stroke 1997;28:491-499.

3. Sellars C, Bowie L, Bagg J, Sweeney MP, Miller H, Tilston J, et al. Risk factors for chest infection in acute stroke: a prospective cohort study. Stroke 2007;38:2284-2291.

4. Leone M, Bouadma L, Bouhemad B, Brissaud O, Dauger S, Gibot S, et al. Hospital-acquired pneumonia in ICU. Anaesth Crit Care Pain Med 2018;37:83-98.

5. Dodek P, Keenan S, Cook D, Heyland D, Jacka M, Hand L, et al. Evidence-based clinical practice guideline for the prevention of ventilator-associated pneumonia. Ann Intern Med 2004; 141:305-313.

6. Kerver AJ, Rommes JH, Mevissen-Verhage EA, Hulstaert PF, Vos A, Verhoef J, et al. Colonization and infection in surgical intensive care patients--a prospective study. Intensive Care Med 1987;13:347-351.

7. Diringer MN, Reaven NL, Funk SE, Uman GC. Elevated body temperature independently contributes to increased length of stay in neurologic intensive care unit patients. Crit Care Med 2004;32:1489-1495.

8. Douds GL, Tadzong B, Agarwal AD, Krishnamurthy S, Lehman EB, Cockroft KM. Influence of Fever and hospital-acquired infection on the incidence of delayed neurological deficit and poor outcome after aneurysmal subarachnoid hemorrhage.
Neurol Res Int 2012;2012:479865.

9. American Thoracic Society. Hospital-acquired pneumonia in adults: diagnosis, assessment of severity, initial antimicrobial therapy, and preventive strategies. A consensus statement, American Thoracic Society, November 1995. Am J Respir Crit Care Med 1996;153(5):1711-1725.

10. Park C, Charalambous LT, Yang Z, Adil SM, Hodges SE, Lee $\mathrm{HJ}$, et al. Inpatient mortality and healthcare resource utilization of nontraumatic intracerebral hemorrhage complications in the US. J Neurosurg 2021;22:1-10.

11. Spalding MC, Cripps MW, Minshall CT. Ventilator-Associated Pneumonia: New Definitions. Crit Care Clin 2017;33:277292.

12. Wang KW, Chen HJ, Lu K, Liliang PC, Huang CK, Tang PL, et al. Pneumonia in patients with severe head injury: incidence, risk factors, and outcomes. J Neurosurg 2013;118: 358-363.

13. LaForce FM. Hospital-acquired gram-negative rod pneumonias: an overview. Am J Med 1981;70:664-669.

14. Alsumrain M, Melillo N, Debari VA, Kirmani J, Moussavi M, Doraiswamy V, et al. Predictors and outcomes of pneumonia in patients with spontaneous intracerebral hemorrhage.J Intensive Care Med 2013;28:118-123.

15. Arnold M, Liesirova K, Broeg-Morvay A, Meisterernst J, Schlager M, Mono ML, et al. Dysphagia in Acute Stroke: Incidence, Burden and Impact on Clinical Outcome. PLoS One 2016;11:e0148424.

16. Martino R, Foley N, Bhogal S, Diamant N, Speechley M, Teasell R. Dysphagia after stroke: incidence, diagnosis, and pulmonary complications. Stroke 2005;36:2756-2763.

17. Langmore SE, Terpenning MS, Schork A, Chen Y, Murray JT, Lopatin D, et al. Predictors of aspiration pneumonia: how important is dysphagia? Dysphagia 1998;13:69-81.

18. Brogan E, Langdon C, Brookes K, Budgeon C, Blacker D. Respiratory infections in acute stroke: nasogastric tubes and immobility are stronger predictors than dysphagia. Dysphagia 2014;29:340-345.

19. Koziel H, Koziel MJ. Pulmonary complications of diabetes mellitus. Pneumonia. Infect Dis Clin North Am 1995;9:65-96.

20. Kornum JB, Thomsen RW, Riis A, Lervang HH, Schønheyder HC, Sørensen HT. Diabetes, glycemic control, and risk of hospitalization with pneumonia: a population-based case-control study. Diabetes Care 2008;31:1541-1545.

21. Costa RD, Baptista JP, Freitas R, Martins PJ. Hospital-acquired pneumonia in a multipurpose intensive care unit: one-year prospective study. Acta Med Port 2019;32:746-753. 